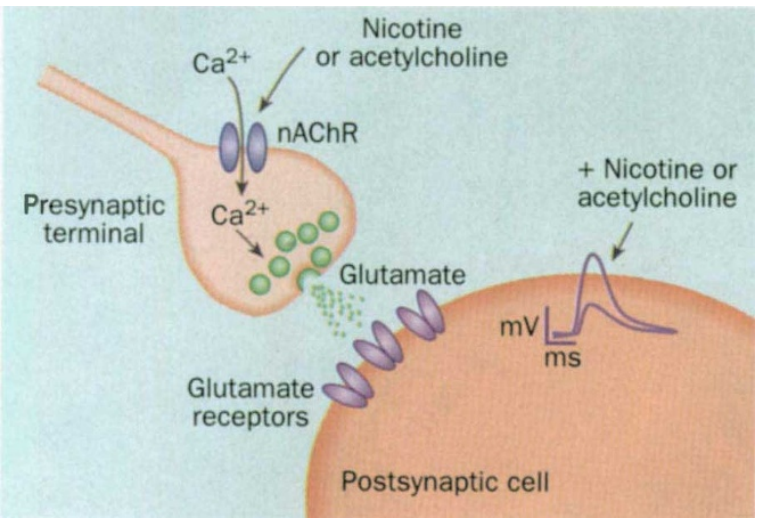

FIG. 2 Low concentrations of nicotine activate nAChRs expressed on presynaptic terminals, and this induces an influx of $\mathrm{Ca}^{2+}$. The subsequent increase in intracellular $\mathrm{Ca}^{2+}$ enhances vesicle fusion and exocytosis. At a synapse (such as the one illustrated here), nicotine enhances vesicular release of the excitatory neurotransmitter glutamate. The inset depicts an increase in the magnitude of the evoked postsynaptic voltage response due to the increased release of glutamate from the presynaptic terminal in the presence of nicotine or acetylcholine ${ }^{12}$. Nicotine-induced $\mathrm{Ca}^{2+}$ influx also enhances the spontaneous release of glutamate, as shown by Gray et al. ${ }^{2}$.

could form homomeric channels in Xenopus oocytes, and that these were blocked by nanomolar concentrations of $\alpha-\mathrm{BGT}^{6-10}$.

Nicotinic nAChRs that include the $\alpha 7$ subunit are highly permeable to calcium $^{8,11}$, indicating that these receptors are ideally suited to induce calcium-sensitive processes such as neurotransmitter release. Although the enhancement of excitatory synaptic transmission by $\alpha$ BGT-sensitive nAChRs has been observed in other systems ${ }^{12,13}$, the involvement of $\alpha 7$ in mediating the presynaptic effects of nicotine seems to be the exception rather than the rule: it is well established that the activation of $\alpha$ BGT-insensitive nAChRs (for example, $\alpha 4 \beta 2$ ) enhances the release of numerous neurotransmitters in several regions of the central nervous system ${ }^{11,14,15}$. Furthermore, these $\alpha / \beta$-type nAChRs also have significant calcium permeability $^{16}$.

Although nicotine can enhance neurotransmitter release in many regions of the central nervous system, it is likely that the activation of presynaptic nAChRs is just one of many steps required for nicotine to alter behaviour or cognition $^{17}$. The cascade of events that follows an initial activation of presynaptic nAChRs most notably includes the entry of calcium as well as the desensitization and, ultimately, inactivation of the nAChRs. Furthermore, all of the nAChRmediated effects depend on the total concentration of the various agonists. Thus, the level of endogenous acetylcholine upon which the exogenously administered nicotine is superimposed will contribute to the net physiological effect.

Even the first cigarette of the day anecdotally renowned for being the best - may deliver nicotine to presynaptic
nAChRs that have already been exposed to endogenous neurotransmitter. In this context, will the exogenous drug further activate the nAChRs and enhance neurotransmitter release as suggested by in vitro analyses? Or is the additional nicotine simply 'excess' agonist which is sufficient to drive presynaptic $\mathrm{nAChRs}$ into the desensitized, non-conducting states? In the latter case, exogenous nicotine would decrease transmission at sites where endogenous acetylcholine release enhances synaptic gain. Furthermore, the effect of nicotine at a given synapse will depend on the acetylcholine-mediated activation and inactivation of the specific nAChRs that are expressed $(\alpha / \beta$ type or $\alpha 7$-type, or both). In the absence of information on the levels and possible sources of acetylcholine, the relative contributions of activation and inactivation of presynaptic nAChRs to the effects of nicotine in the central nervous system is still beyond reach. Nevertheless, the definitive demonstration by Gray et al. ${ }^{2}$ that nicotine potently enhances transmission in the hippocampus is unquestionably an important step towards recalling nicotine as a powerful modulator of memory.

Daniel S. McGehee is in the Department of Anesthesia and Critical Care, University of Chicago, Whitman Laboratory, 915 East 57th Street, Chicago, Illinois 60637, USA. Lorna W. Role is in the Department of Anatomy and Cell Biology, Center for Neurobiology and Behavior, Columbia University, 722 West 168th Street, New York, New York 10032, USA.

\title{
Clever use of light and unparalleled flow
}
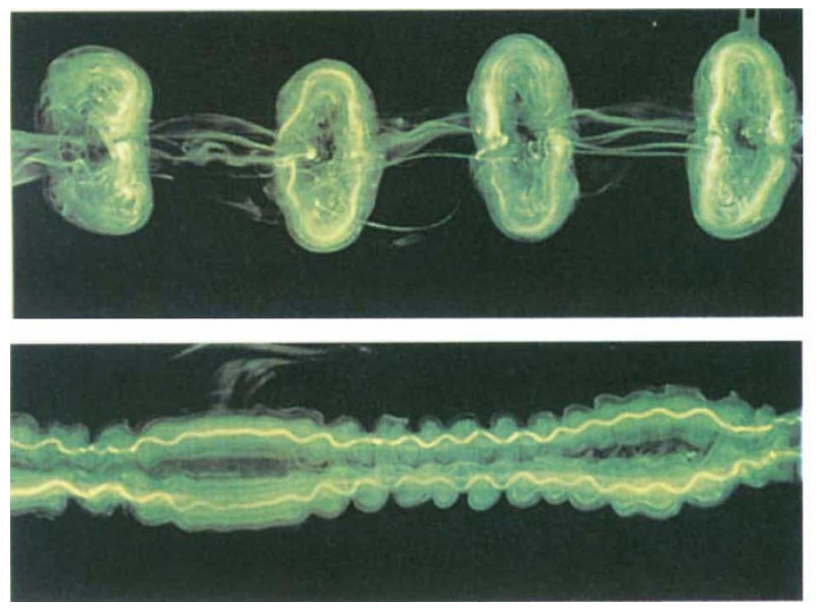

THESE striking images are from this year's Gallery of Fluid Motion (Phys. Fluids 8, S1-S12; 1996).

On the left, a fluorescent dye traces the long- and shortwavelength instabilities of a pair of counter-rotating vortices. In the upper panel, the two initially straight, parallel vortices have broken up and reconnected to form rings - an effect that has been predicted but never produced before (T. Leweke \& C. H. K. Williamson, Cornell Univ.).
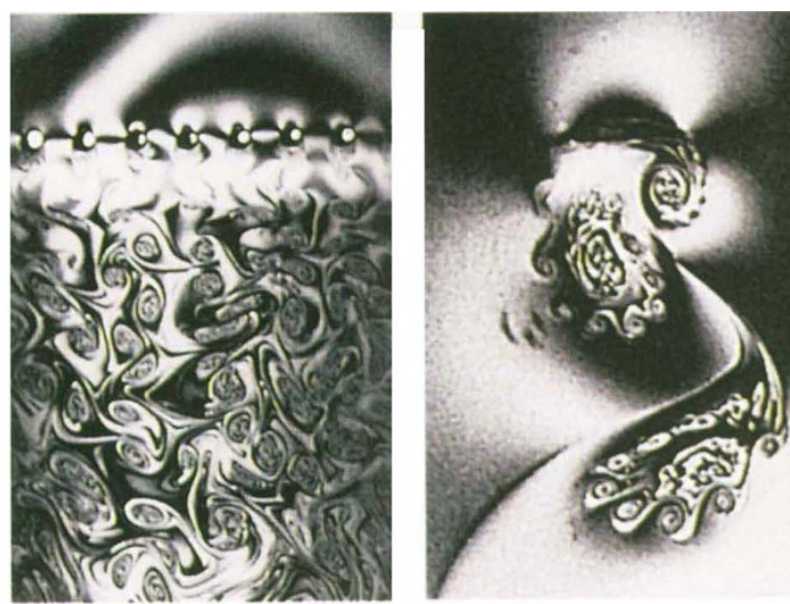

On the right, a soap film is suspended between two vertical nylon wires, and flows at a terminal velocity of two or three $\mathrm{m} \mathrm{s}^{-1}$ down past an array of comb teeth and a knife (far right), whose sharper edges produce particularly fine structure that may be useful in generating turbulence. Interference fringes show up the thickness variations in the vortices (M. A. Rutgers, X.-L. Wu \& W. I. Goldburg, Univ. Pittsburgh). Stephen Battersby 